\title{
WHY PATIENTS MISS FOLLOW-UP APPOINTMENTS: A PROSPECTIVE CONTROL-MATCHED STUDY
}

\author{
G. Van der Meer and J.W. Loock
}

\section{Abstract:}

Objectives: To investigate missed appointments in a South African tertiary hospital.

Study Design: Prospective, descriptive series with controls.

Setting: T he ENT/Oncology clinic at Tygerberg Academic Hospital, South Africa. Subjects: 305 patients with a head and neck malignancy who had follow-up appointments over 4 consecutive months between June and September 2006 . A control group of 31 patients who attended the clinic was recruited in September 2006.

Method: Analysis of the clinic attendance statistics to identify patients who missed follow-up appointments followed by a file review and interview of these patients. The results were compared with a control group. Outcome measures: 1) Incidence rate of failure to attend follow-up. 2) Causative factors

Results: $51(17 \%)$ booked patients missed their appointments. Non-attenders were most likely to miss their follow-up between 6 and 12 months (17/31) after treatment. No correlations were found between diagnosis, disease stage and missed appointments.

Reasons include: transport (19 responses), ill-health (6) and financial constraints (5). State transport was unavailable to almost twothirds of the responders who cited transport as a problem.

Conclusions: The $17 \%$ missed appointment rate is largely due to transport constraints. The commonest time for patients to miss appointments is the 6-12 month follow-up period. The authors seek to identify patients at risk of missed appointments and suggest interventions to decrease this incidence.

Keywords: Cancer, Appointments, Transport, Follow-up

\section{Introduction:}

Missed appointments remain a ubiquitous part of any health care system. The implications of missed appointments to health systems include:

1) Financial Impact: 6 million missed appointments in the United Kingdom in 1997 cost an estimated $€ 300$ million (1).

2) Underutilization of facilities (2).

3) Neglect of treatable conditions leads to an increased demand at a later stage (3).

The implications for patients include: 1) Delayed treatment of potential complications of the cancer treatment. 2) Missing early signs of malignancy recurrence. 3) Long delays to a new follow-up date. 4) Possible permanent loss to follow-up as the patient disappears from clinic records.

In the developed world, studies have been conducted in an attempt to elucidate the causes and to diminish the incidence of missed appointments. South Africa has unique pressures with regard to health care attendance, including pervasive poverty, long distances and health care system constraints which makes comparison with developed countries difficult

Figures for South Africa are not readily available, but in the UK, the incidence of non-arrival at surgical and medical clinics (no data available for ENT oncology clinics) ranges between $10 \%$ and $30 \%$ with a mean of $12 \%{ }^{2}$ Some of the reasons include: forgetfulness, fear of treatment, clerical errors and improved health. Murdock et al suggest that patient apathy plays a large role in the current burden of missed appointments. Most investigations into the factors influencing missed appointments are limited by the poor response

\footnotetext{
Correspondance to: G van der Meer , Department of Otorhinolaryngology, Tygerberg Hospital / University of Stellenbosch Faculty of Health Sciences, Francie van $\mathrm{Zijl}$ Dr Parow, 7505, South Africa, Email: graeme@ $a$ prometics.com, Tel: +27 (0)21-9384154, Fax: +27 (0)21 - 9389470

Department of Otorhinolaryngology, Tygerberg Hospital / University of Stellenbosch Faculty of Health Sciences

Cape Town, South Africa
}

rates, typically $30-40 \%$ (4), yet it was found that telephonic interviews achieved a response rate of $93 \%$ (2) One of the cornerstones of cancer management is regular follow-up, not only to alleviate any side-effects of treatment, but also to screen for recurrence. The study aimed to evaluate the rate of, and factors leading to, missed appointments.

\section{Ethical Approval}

This study received ethical approval from the Committee for Human Research at Stellenbosch University / Tygerberg Hospital and conformed to the principles embodied in the Declaration of Helsinki.

\section{Patients and Methods:}

This study included all patients who missed their follow-up appointments at the Tygerberg Combined ENT Oncology Clinic over a period of 4 consecutive months from June to September 2006. A list of patients who attended and missed their follow-up appointment was obtained after every clinic. The files of those patients who missed the clinic were reviewed and all the available contact details were noted as well as the patients' demographic information. Patients were interviewed telephonically or, failing that, personally when they returned after being contacted through reminder letters sent by the clinic. The study subjects, after giving informed consent, completed an anonymous form which included: general demographics, diagnosis, treatment type, duration of treatment, experience of treatment, follow-up time, reasons for missing the appointment and open-ended questions about ways to improve service delivery. Patients could supply more than one reason. New dates for follow-up visits were also arranged at the interview.

A control group of 31 consecutive patients who attended the same clinic in September 2006 was recruited to the study and completed a similar questionnaire. Demographic breakdown of the patients who could not be 
contacted was also undertaken.

\section{Results:}

Of the 305 patient bookings over the duration of the study, 254 (83\%) of patients kept their appointments at the clinics, while $51(17 \%)$ missed appointments. Of the 51 patients, 8 (3\%) had died, 30 (10\%) were interviewed and it was not possible to establish contact with the remaining 13 (4\%).

A cohort of 30 patients who did attend was created and contrasted with the first group. The groups were found to be similar with regard to age (56.8 vs 58.9 years) and gender ( $88 \%$ vs $87 \%$ male). The group who attended the clinics had an average of 8.26 years of schooling, compared with the 7.2 years of the non-attenders, which was not statistically significant. $(p=0.12)$ Although only $67 \%$ of non-attenders realized the importance of attending the clinics, compared with $84 \%$ of attenders, this was not statistically significant. No correlations were found between diagnosis, disease stage and missed appointments.

Analysis of the follow-up times showed a striking pattern: The non-attenders were most prone to do so between 6 and 12 months after treatment with 17 cases (57\%). (Table 1) The commonest reasons cited for nonattendance were: transport (19 cases), health reasons (6 cases) and finances (5 cases). All the reasons are listed in Table 2.

Analysis of the subset who listed transport as a factor revealed that state transport was only available to 7 (37\%) with an equal number having no available transport and the remaining 5 (26\%) having been denied access to the transport due to space constraints.

Of the 13 unreachable patients, there were 11 males and 2 females with an average age of 52.8 years. Of these, 11 (85\%) stayed in rural areas, 10 (77\%) stayed more than $150 \mathrm{~km}$ away from the follow up clinic. 7 patients (54\%) lived on farms, and 6 patients (46\%) lived on farms in excess of $150 \mathrm{~km}$ away.

\section{Table 1:Timing of Missed Appointments}

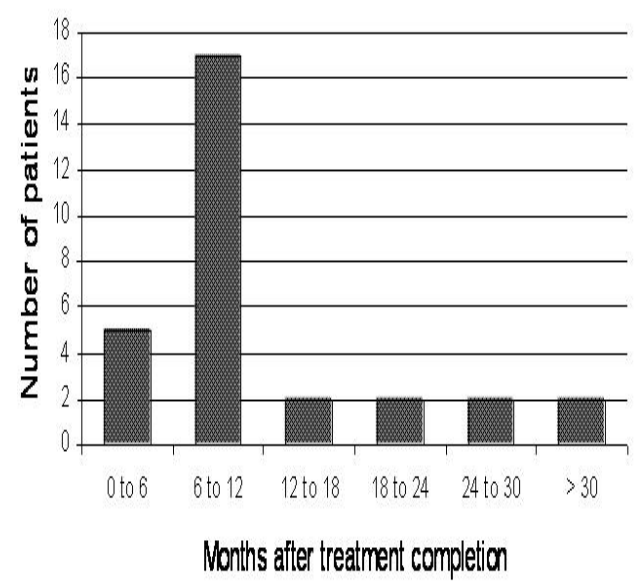

\section{Table 2: Reasons for Missed Appointments}

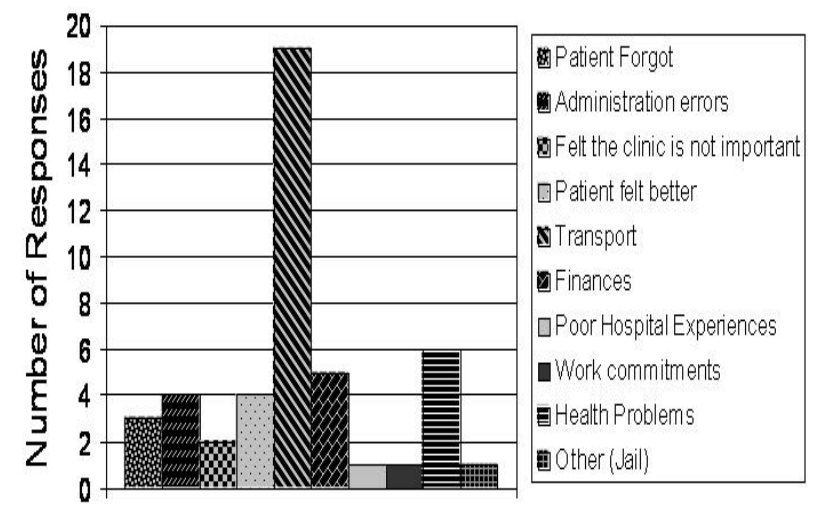

Reason

\section{Discussion:}

This study demonstrates a 1 in 6 incidence of nonattendance of the oncology follow-up clinics, which is similar to international findings for general clinics. This is where the similarity ends as South Africa is faced with unique challenges in the form of limited health care, long distances, poor education and limited methods of distance communication.

As a reflection on all clinics in the South African health system, it is probably an underestimation, as special care is taken with each cancer patient upon diagnosis to ensure correct contact details and adherence to follow-up. Each patient is counselled by a dedicated social worker, and this, coupled with the understood severity of their disease, may encourage better follow-up.

There was no statistically significant difference in education levels and while non-attenders demonstrated a trend to 1 year less schooling than those who attended the clinics, education level does not seem to play a prominent role in missing appointments.

A concern demonstrated by this study is that the majority of missed appointments occurred in the 6 to 12 month follow-up period, which is considered a critical time for the early recognition of oncological treatment failure, as the side-effects of radiotherapy resolve and may unmask recurrent or residual disease.

The main reason cited for missing the follow-up clinic is lack of transport, and the unavailability of state transport to $63 \%$ of those with transport constraints is a sad indictment on the current provisions of health care for all South Africans. Again, cancer survivors are given preference on the buses and have an easier path to securing state transport, but they remain dependent on space availability. It can be assumed that non-cancer patients who are not afforded transport preference are 
more adversely affected.

A decrease in both the hospital and transport tariffs at the time of this study contributed to far fewer patients listing cost as a barrier to health care than was expected.

Contrary to international literature, patient apathy did not seem to play a significant role in missed appointments. The authors made an effort to counsel the study subjects to answer the anonymous questionnaires truthfully and without feeling the need to hide reasons, and the results are seen to be an accurate reflection of the underlying issues.

The authors sought to identify those patients who seemed to be most susceptible to missing appointments and these include:

1) Patients in the 6-12 month follow-up period.

2) Those living on farms especially more than $150 \mathrm{~km}$ away from the hospital.

3) Patients reliant on space-limited state transport services.

Furthermore, the authors suggest that interventions which could limit the prevalence of non-attendance include:

1) At the first visit: Identifying patients at risk by discussing the potential for missed appointments and placing emphasis on obtaining patient contact details and systems of pursuit.

2) Providing hospital contact details and methods of contacting the clinic so that the patient can either reschedule a future appointment or get a new date after a missed appointment.

3) A booking notification system whereby all patients who miss an appointment get highlighted and contacted by the clinic.

4) Transport facility evaluation and improvement by the Health Department, specifically regarding increasing the amount of space available on buses and reaching more rural areas.

5) Provision of rural outreach follow-up oncology clinics.

6) Close liaison with rural health facilities would be beneficial for the monitoring of treatment outcomes and, if a patient dies, to rebook another patient in his or her place at the clinic.

Further studies in this field are envisaged and will include an audit of the improvements made to the followup system.

\section{What this study adds:}

Missed appointments have been investigated in first world countries, but these results have poor application to developing countries. No research has been published that investigates the plight of patients who miss health care appointments in South Africa.

\section{Policy Implications:}

This study identifies the main causative factor of missed appointments in South Africa, namely transport constraints, and highlights that patients are most likely to miss their appointments between 6 and 12 months after initial treatment. Based on these results, the authors suggest interventions and changes to the current policy for improved health care provision.

\section{References:}

1) Committee of Public Accounts. $42^{\text {nd }}$ Report. National Health Service in England and Wales. London; Stationery Office.

Murdock $A$ et al. Why do patients not keep their appointments? $J$ R Soc Med, 2002;95:284-6

3) Andrews R, et al. Understanding non-attendance in pediatric outpatient clinics. Arch Dis Child 1990; 65: 195

4) Jones RB, et al. Reducing non attendance in an outpatient clinic. Pub Health 1998;102:385-91 